Women with prenatal diagnosis were not elderly, and most had previous pregnancies without abnormalities.

An important number of CNS malformations are associated with genes or chromosomal syndromes (24\%), and the most frequently isolated cases correspond to NTD (42.2\%), it believes that this group can be prevented by the intake of preconception folic acid.

\section{PO-0452 HYPOXIC-ISCHAEMIC ENCEPHALOPATHY AND PERINATAL INFECTION: A PILOT STUDY}

${ }^{1}$ A Cilla, ${ }^{2}$ J Arnaez, ${ }^{2} \mathrm{~J}$ Suarez, ${ }^{3} \mathrm{G}$ Megias, ${ }^{4} \mathrm{M}$ Cabrerizo, ${ }^{5} \mathrm{~A}$ García-Álix. ${ }^{1}$ Pediatrics, Hospital Universitario de Burgos, Burgos, Spain; ${ }^{2}$ Neonatology Pediatrics, Hospital Universitario de Burgos, Burgos, Spain; ${ }^{3}$ Microbiology, Hospital Universitario de Burgos, Burgos, Spain; ${ }^{4}$ Microbiology, Centro Nacional de Microbiología Instituto de Salud Carlos III, Madrid, Spain; ${ }^{5}$ Neonatology, Hospital Sant Joan de Deu, Barcelona, Spain

\subsection{6/archdischild-2014-307384.1093}

Background Recent studies suggest a synergic effect of infection and hypoxia-ischemia in the causation of perinatal brain damage. Although an infection screening is warranted in cases of hypoxic-ischaemic encephalopathy (HIE), whether this screening should include more infrequent pathogens like neurotropic viruses is controversial.

Objectives To evaluate the importance of perinatal infection in HIE, focusing on neurotropic viruses.

Methods Prospective study including infants above 35 weeks gestation diagnosed of moderate or severe HIE in Burgos University Hospital during the period October 2011-2013. Antenatal and perinatal data were recorded, as well as details of the clinical course in the neonatal period. Serological studies were performed to the mother during pregnancy. Bacterial blood and cerebrospinal cultures, as well as viral tests (Cytomegalovirus, Epstein-Barr virus, Human Herpes virus, Enterovirus, Parechovirus) in cerebrospinal fluid were performed to the newborns at birth.

Results 12 newborns were included in the study. There were no confirmed cases of viral infection. There was a case of bacterial early onset sepsis and three cases of suspected sepsis due to clini$\mathrm{cal}$ and/or analytical signs, but with negative cultures. An elevation of the $\mathrm{C}$ reactive protein (CRP) levels was the sole cause of suspicion in two of these cases.

Conclusions Our results confirm that an infection screening is important in HIE. These pilot results would not support universal screening for viral infection in cases of HIE.

\section{PO-0453 MATURATIONAL CHANGES IN CORTICAL FOLDING IN EXTREMELY PRETERM INFANTS}

${ }^{1} \mathrm{NHP}$ Claessens, ${ }^{1} \mathrm{KJ}$ Kersbergen, ${ }^{2} \mathrm{~F}$ Leroy, ${ }^{3} \mathrm{P}$ Moeskops, ${ }^{3} \mathrm{I}$ Isgum, ${ }^{3} \mathrm{MA}$ Viergever, ${ }^{1} \mathrm{~F}$ Groenendaal, ${ }^{1} \mathrm{LS}$ De Vries, ${ }^{2} \mathrm{~J}$ Dubois, ${ }^{4} \mathrm{M}$ MNL Benders. ${ }^{1}$ Department of Perinatology, Wilhemina Children's Hospital University Medical Center Utrecht, Utrecht, Netherlands; ${ }^{2}$ INSERM Cognitive Neuroimaging Unit U992, CEA DSV I2BM Neurospin Center, Gif Sur Yvette, France; ${ }^{3}$ Image Sciences Institute, University Medical Center Utrecht, Utrecht, Netherlands; ${ }^{4}$ Centre for Developing Brain King's College London UK and Department of Perinatology, Wilhemina Children's Hospital University Medical Center Utrecht, Utrecht, Netherlands

10.1136/archdischild-2014-307384.1094

Background and aim Our aim was to analyse the development of cortical morphology in preterm infants, as alterations in cortical folding affect functional development.
Methods MRI was performed at 30 and 40 weeks corrected age in 19 preterm born infants (gestational age (GA) 24.71-27.86 wks, 11 males). After automatic brain tissue segmentation, ${ }^{1}$ a $3 \mathrm{D}$ reconstruction of the inner cortical surface was computed and cortical sulci were labelled with Brainvisa software. ${ }^{2}$ We measured global sulcal index ( $\mathrm{SI}=$ folded/unfolded surface areas), and per sulcus surface area $\left(\mathrm{SA}, \mathrm{mm}^{2}\right)$ and mean geodesic depth (MGD, mm), all corrected for scan-age.

Results In 10 weeks, SI increased from 0.18 to 1.08 (6 times, 40 wks: right $>$ left). Central sulcus, lateral fissure, and insula increased more in SA (resp. 2.3, 2.6, 1.7-times) than in MGD (resp.1.2, 1.1, 1.3-times). The superior temporal sulcus (STS) expanded in SA with factor 16.3 left and 12.6 right (30 wks: right > left), and factor 1.6 in MGD (30 and 40 wks: right > left). White matter injury in these infants (9 IVH,1 PVHI, 4 treated for PHVD) or GA did not significantly influence cortical morphology changes.

Conclusion Over this short period, cortical folding is immense in preterm newborns, and shows inter-hemispherical asymmetries. Sulci increased more in surface area than in depth, STS showed the largest increase. The influence of brain injury on cortical morphology needs to be elucidated in a larger cohort.

\section{REFERENCES}

1 Chita S, et al. Medical Imaging (2013)

2 Perrot M, et al. Medical Image Analysis (2011)

\section{P0-0454 IMPACT OF SYSTEMATIC PAIN AND SEDATION MANAGEMENT ON OUTCOME OF VERY LOW BIRTH WEIGHT INFANTS}

${ }^{1} \mathrm{P}$ Deindl, ${ }^{2} \mathrm{R}$ Fuiko, ${ }^{3} \mathrm{~T}$ Waldhoer, ${ }^{4} \mathrm{G}$ Kappler, ${ }^{2} \mathrm{~L}$ Unterasinger, ${ }^{2} \mathrm{~V}$ Giordano, ${ }^{2} \mathrm{~T}$ Werther, ${ }^{2} \mathrm{C}$ Czaba, ${ }^{2}$ Geroldinger, ${ }^{2} \mathrm{~A}$ Berger, ${ }^{2} \mathrm{M}$ Olischar. ${ }^{1}$ Department of Neonatology and Pediatric Intensive Care Medicine, University Medical Center Hamburg-Eppendorf, Hamburg, Germany; ${ }^{2}$ Department of Pediatrics and Adolescent Medicine Division of Neonatology Pediatric Intensive Care and Neuropediatrics, Medical University of Vienna, Vienna, Austria; ${ }^{3}$ Department of Epidemiology Center for Public Health, Medical University of Vienna, Vienna, Austria; ${ }^{4}$ Faculty of Psychology Institute for Applied Psychology: Health Development Enhancement Intervention, University of Vienna, Vienna, Austria

\subsection{6/archdischild-2014-307384.1095}

Background and objectives We retrospectively compared shortterm and neurodevelopmental outcome of very low birth weight infants (VLBWI) before $(\mathrm{n}=84)$ and after implementation $(\mathrm{n}=$ 69 ) of a protocol for the management of neonatal pain and sedation.

Methods Opiate exposure, time on mechanical ventilation, inotropic support, details on nutritional aspects, and growth were compared between baseline and after protocol implementation. Infants were evaluated at 12 months corrected age using standardised neurologic examination and Bayley Scales of Infant Development-II.

Results Cumulative mean \pm SD opiate dose (baseline dose of 14 $\pm 39 \mathrm{mg} / \mathrm{kg}$ vs. intervention group dose of $84 \pm 222 \mathrm{mg} / \mathrm{kg}$ morphine equivalents; $\mathrm{p}<0$. 0001) increased after implementation. Time on mechanical ventilation, inotropic support, time on parenteral nutrition, growth, and length of stay were similar before and after implementation. There were no differences in neurodevelopmental outcome variables before and after intervention (MDI: $85 \pm 14$ vs. $84 \pm 16, p=0.6$; PDI: $87 \pm 19$ vs. $83 \pm 19, \mathrm{p}=0.2$; BRS: $74 \pm 27$ vs. $68 \pm 32, \mathrm{p}=0.2$ ). Multiple linear regression analysis identified opiate exposure as a possible risk factor for lower MDI (estimate $=-0.15 ; \mathrm{p}=0.004$ ) 
and Behaviour Rating Scale (BRS) scores (estimate $=-0.39 ; \mathrm{p}=$ 0.012).

Conclusions Implementation of a neonatal pain and sedation protocol results in an increase in opiate prescription without affecting short-term outcome and neurodevelopmental performance of VLBWI at 12 months corrected age.

\section{P0-0455 RESISTIVE INDEX (RI) OF CEREBRAL ARTERIES IN VERY PRETERM INFANTS: REFERENCE VALUES AND IMPACT OF PATENT DUCTUS ARTERIOSUS}

${ }^{1} \mathrm{GM}$ Ecury-Goossen, 'MMA Raets, ${ }^{2} \mathrm{FA}$ Camfferman, ${ }^{3} \mathrm{~J}$ van Rosmalen, 'IKM Reiss, ${ }^{4} \mathrm{P}$ Govaert, 'J Dudink. 'Department of Pediatrics, Division of Neonatology, Erasmus MC-Sophia Children's Hospital, Rotterdam, Netherlands; ${ }^{2}$ Department of Pediatrics Division of Neonatology, UZ Brussel, Rotterdam, Netherlands; ${ }^{3}$ Department of Biostatistics, Erasmus MC, Rotterdam, Netherlands; ${ }^{4}$ Department of Pediatrics, Koningin Paola Children's Hospital, Antwerp, Belgium

\subsection{6/archdischild-2014-307384.1096}

Background and aims We describe resistive index (RI) values in various cerebral arteries in a cohort of preterm infants born at $<29$ weeks gestation. We also aim to compare RI in various intracranial arteries, and to assess the relationship between RI and hemodynamically significant patent ductus arteriosus (PDA). Methods Preterm infants admitted to our NICU between May 2010 and January 2013 were enrolled. RI of internal carotid arteries, basilar artery, anterior cerebral artery, pial and striatal arteries was obtained in the first three days of life and weekly after that until discharge or death.

Results 771 examinations were performed in 235 infants. RI differed depending on which artery was insonated. RI was negatively correlated with measured time averaged velocity, except in the basilar artery. There was a significant difference in RI when comparing left (mean 0.86) to right sided (0.84) internal carotid artery $(\mathrm{p}=0.023)$. RI was lower in patients without PDA compared to patients with hemodynamically significant PDA, though this difference was small and not statistically significant in all arteries. There was no difference in pre- and postligation RI in patients who underwent PDA ligation. RI was not related to gestational age, postnatal age, SNAPPE II score or gender.

Conclusions For accurate follow-up and comparison of RI, it is important to examine the same artery. No definitive cut-off value for RI indicative for the presence of a hemodynamically significant PDA was found. Cerebral artery RI may have limited value as a clinical tool in the very preterm infant.

\section{PO-0456 MEASURING CEREBRAL AUTOREGULATION. A COMPARISON OF FREQUENCY - AND TIME DOMAIN ANALYSIS}

VR Eriksen, GH Hahn, G Greisen. Department of Neonatology, Rigshospitalet Copenhagen University Hospital, Copenhagen, Denmark

\subsection{6/archdischild-2014-307384.1097}

Background and aims Cerebral autoregulation (CA) is described by the relation between mean arterial blood pressure and cerebral blood flow. The aim of this study was to compare frequency - and time domain analysis - two conventional methods used to describe CA.

Methods We measured cerebral oxygenation (as a surrogate for cerebral blood flow) and mean arterial blood pressure in 60 preterm infants in the first day of life. Coherence and gain were calculated by means of frequency domain analysis. The cerebral oxyimetry index (COx) and the regression coefficient were calculated in the time domain. For both calculations $5 \mathrm{~min}$ windows were applied in 4-17 consecutive $10 \mathrm{~min}$ epochs of good quality signal in each infant. Analysis of variance was used to compare the intra-infant and inter-infant variation.

Results Discrimination among the infants was less good for coherence than for $\mathrm{COx}(\mathrm{F}=3.4$ vs. 8.8). The correlation between coherence and COx was poor (Pearson's $r=0.215$, $\mathrm{p}=0.097)$. Applying conventional thresholds for 'impaired' autoregulation resulted in different classifications (Chi2 $=3.78$, $\mathrm{p}=0.052$ ). In one extreme case, gain was $0.68 \mathrm{microM} / \mathrm{mmHg}$ - the highest of all the infants - while the regression coeffficient of the time-domain analysis was $-0.33 \mathrm{microM} / \mathrm{mmHg}$ - the lowest of all. This was due to cerebral oxygenation and arterial blood pressure being in counterphase.

Conclusions Time domain analysis gave better discrimination among infants, suggesting better precision. Also, a high gain and high coherence may arise spuriously when cerebral oxygenation decreases as blood pressure increases.

\section{PO-0457 ASSOCIATION BETWEEN EARLY VISUAL MOTION PERCEPTION IN VERY PRETERM CHILDREN AND NEURODEVELOPMENT AT 2.5 YEARS}

${ }^{1} \mathrm{Y}$ Fredriksson Kaul, ${ }^{2} \mathrm{~K}$ Rosander, ${ }^{2} \mathrm{C}$ von Hofsten, ${ }^{3} \mathrm{~B}$ Böhm, ${ }^{1} \mathrm{~K}$ Strand Brodd, 'L Hellström Westas. 'Department of Women's and Children's Health, Uppsala University, Uppsala, Sweden; ${ }^{2}$ Department of Psychology, Uppsala University, Uppsala, Sweden; ${ }^{3}$ Department of Women's and Children's Health, Karolinska Institute, Stockholm, Sweden

\subsection{6/archdischild-2014-307384.1098}

Introduction Young infants need visual motion perception to understand their environment. Gaze reflects the ability to track moving objects, and includes smooth pursuit (SP) eye movements, saccades and head movements.

Aim To investigate if early visual tracking ability in children born very preterm (VPT) is associated with later neurodevelopment.

Method The VPT infants $(n=68)$ had mean (SD) gestational age of 28.3 (2.6) weeks and birth weight 1154 (365) g. Ability to track moving objects was recorded at 4 months corrected age (CA) using a system combining electro-oculography and cameras recording head movements. Gaze and proportion of SP were measured. Tracking ability corresponding to at least the 10th percentile of a term born control group was defined as normal, and served as cut off for VPT infants. Neurodevelopment was assessed in VPT infants at 2.5 years (Bayley Scales of Infant Development III, BSID III).

Results At 4 months $37 \%$ and 25\% of VPT infants had subnormal SP and Gaze, respectively. The table shows group differences in BSID III subscale scores for Gaze and SP, between children with normal compared to subnormal early visual tracking ability.

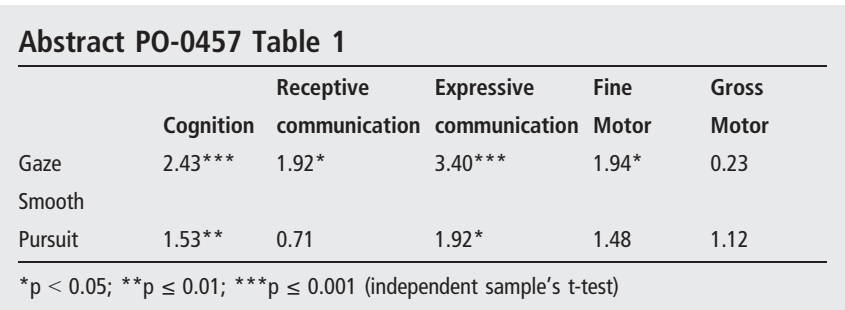

